\title{
External Uterine Contractions Signal Analysis in Relation to Labor Progression and Dystocia
}

\author{
H. Gonçalves ${ }^{1}$, P. Pinto ${ }^{2,3}$, D. Ayres-de-Campos ${ }^{1,3,4,5}$, and J. Bernardes ${ }^{1,3,4,5}$ \\ ${ }^{1}$ Center for Research in Health Technologies and Information Systems (CINTESIS), \\ Faculty of Medicine, University of Porto, Porto, Portugal \\ ${ }^{2}$ Hospital Dr Nélio Mendonça, EPE, Funchal, Portugal \\ ${ }^{3}$ Department of Obstetrics and Gynecology, Faculty of Medicine, University of Porto, Porto, Portugal \\ ${ }^{4}$ Department of Obstetrics and Gynecology, São João Hospital, Porto, Portugal \\ ${ }^{5}$ INEB - Institute of Biomedical Engineering, Porto, Portugal
}

\begin{abstract}
Labor dystocia is a major cause of operative delivery, which is associated with increased risks for both mother and fetus. We assessed linear and non-linear dynamics of external uterine contraction signals, in relation to labor progression and dystocia. Linear time domain, spectral and entropy methods were used to analyze external uterine contraction recordings obtained during the last two hours of labor, in 28 cases with normal and 27 cases with operative deliveries (forceps, vacuum or caesarean). Progression of labor was associated with a statistically significant increase in most linear time domain and spectral indices, both in normal and operative deliveries, whereas most entropy indices increased in normal deliveries, but did not change in operative deliveries. On the other hand, when compared with normal births, operative deliveries were associated with significantly increased entropy indices before the last hour of labor and significantly decreased (a probably associated) sympatho-vagal balance in the last hour of labor. Linear and non-linear analysis of external uterine contraction recordings may provide useful physiopathological and clinical information on the progression of labor and the diagnosis of dystocia.
\end{abstract}

Keywords-Dystocia, External uterine contractions, Cardiotocography, Spectral analysis, entropy.

\section{INTRODUCTION}

There have been several attempts in the last decades to improve the evaluation of labor progression and to predict and manage dystocia, a major cause of maternal and fetal trauma [1]. Among these attempts was the development of algorithms for the analysis of the uterine activity using intrauterine manometry, namely with Caldeyro Barcia and Alexandria units [2]. Spectral and entropy analysis of uterine electromyography (EMG) signals have also been developed using prototype research equipment [3]. However, none of these methods has yet proved effective for routine clinical use.

Uterine contractions (UC) signals are routinely acquired in clinical practice by internal or external tocography [4]. They provide important information for the early detection of preterm labor and abnormal labor progress or dystocia.
However, research on UC analysis is encompassed by a lack of international guidelines on the interpretation of basic signal features, such as those existent for fetal heart rate signals [5-7].

Internal tocography is considered a very accurate method for UC monitoring [8], but it is an invasive technique that increases the risk of infection and requires previous rupture of the fetal membranes [9]. Moreover, internal tocodynamometry during induced or augmented labor, when compared with external monitoring, has not been shown to reduce the rate of operative deliveries or adverse neonatal outcomes [4]. Therefore, the development of efficient methods for the analysis of the external UC signal is an important research challenge, and one that has a great potential for applicability in routine clinical practice. Some interesting progresses with experimental methods for EMG recordings have also been reported, but this technology has still not entered routine clinical practice.

In this paper, we assess linear and non-linear dynamics of UC recordings, in relation with labor progression and dystocia, using external tocodynamometry (ETOCO), obtained with conventional fetal monitors used in routine clinical practice. We explore signal characteristics not only related to UC but also to other characteristics of uterine activity recordings using ETOCO, such as oscillations related to maternal and fetal movements [2].

\section{MATERIALS AND METHODS}

A set of $55 \mathrm{UC}$ recordings were acquired, obtained in the same number of women, during the last hours of labor, consecutively selected among UC recordings, and with a mean duration of $328 \pm 179 \mathrm{~min}$. The dataset was divided in two groups according to the type of delivery: 28 cases of normal delivery; and 27 cases of operative delivery (forceps, vacuum or caesarean). The main perinatal characteristics of the cases included in the study are presented in Table 1 , corresponding to term pregnancies, during labor, with epidural analgesia and favorable outcomes. 
Table 1 Main maternal and fetal characteristics of the normal and operative delivery groups included in the study.

\begin{tabular}{lccc}
\hline Item & $\begin{array}{c}\text { Normal } \\
(\mathrm{n}=28)\end{array}$ & $\begin{array}{c}\text { Operative } \\
(\mathrm{n}=27)\end{array}$ & $\mathrm{P}$ \\
\hline Maternal data, mean (SD) & & & \\
Age (years) & $28.4(4.8)$ & $27.6(5.7)$ & 0.710 \\
Parity & $0.5(0.6)$ & $0.1(0.3)$ & $\mathbf{0 . 0 0 8}$ \\
Gestational age (weeks) & $39.6(1.0)$ & $39.9(1.1)$ & 0.203 \\
Delivery, n (\%) & & & \\
Normal & $28(100.0 \%)$ & $0(0.0 \%)$ & \\
Operative (forceps) & $0(0.0 \%)$ & $9(33.3 \%)$ & \\
Operative (vacuum) & $0(0.0 \%)$ & $12(44.4 \%)$ & \\
Operative (caesarean) & $0(0.0 \%)$ & $6(22.2 \%)$ & \\
Epidural analgesia, $\mathrm{n}(\%)$ & $28(100 \%)$ & $27(100 \%)$ & - \\
Newborn data, mean (SD) & & & \\
Birthweight (grams) & $3233(309)$ & $3266(351)$ & 0.730 \\
1 minute Apgar score & $9.3(0.4)$ & $9.2(0.7)$ & 0.774 \\
5 minute Apgar score & $9.9(0.3)$ & $9.8(0.4)$ & 0.210 \\
Umbilical artery blood pH & $7.25(0.07)$ & $7.23(0.07)$ & 0.304 \\
Gender, n (\%) & & & 0.130 \\
Males & $13(46.4 \%)$ & $18(66.7 \%)$ & \\
Females & $15(53.6 \%)$ & $9(33.3 \%)$ & \\
\hline & & & \\
\hline
\end{tabular}

UC signal was acquired through a conventional external tochodynamometer, applied on the maternal abdomen, linked to a conventional STAN $₫ 31$ fetal monitor (Neoventa, Gothemburg, Sweden). The UC signal was subsequently exported from the STAN monitor at a sampling rate of $4 \mathrm{~Hz}$, via its $\mathrm{RS} 232$ port, into the Omniview-SisPorto ${ }^{\circledR}$ 3.5 program (Speculum, Lisbon, Portugal) [10]. The hour before the last $\left(\mathrm{H}_{1}\right)$ and the last $\left(\mathrm{H}_{2}\right)$ hours of each recording were considered for further processing, where each hour was divided in segments with 5-min length. Each segment was analysed for each case, using time and frequency domain linear, and nonlinear indices, briefly described in the following.

For time domain linear analysis, the following indices were calculated: mean UC (mUC), standard deviation of UC (sdUC), long-term irregularity index (LTI), Delta UC $(\Delta)$, short-term variation (STV) and interval index (II). All but II reflect gross changes in UC average and variability, whereas II assesses short-term UC variability taking into account long-term variability [11]. The frequency domain analysis was based on the nonparametric estimation of the spectrum, according to a procedure previously described in detail [11]. Despite the lack of guidelines for UC spectral analysis, a visual interpretation of the spectral analysis suggested the use of the same spectral bands as in heart rate (HR) analysis. The following frequency bands were considered: very low frequency (VLF) at $0-0.04 \mathrm{~Hz}$; low frequency (LF) at $0.04-0.15 \mathrm{~Hz}$; and high frequency (HF) at $0.15-0.40 \mathrm{~Hz}$. Under the scope of HR variability analysis, LF and HF are mainly associated with activity of the sympathetic and parasympathetic systems, respectively [12]. $\mathrm{LF}_{\text {norm }}$ and $\mathrm{HF}_{\text {norm }}$ were also computed by normalizing each absolute value by (TP-VLF). The LF/HF index, which reflects the balance between the autonomic nervous system branches, as well as total power (TP, i.e., all the spectrum area), were also considered. For non-linear analysis, approximate entropy (ApEn) [13], and sample entropy (SampEn) [14] were calculated, considering values 0.1 SD, $0.15 \mathrm{SD}$ and $0.2 \mathrm{SD}$ for $\mathrm{r}$ and value 2 for $\mathrm{m}$ [15], while $\mathrm{N}$ was 1200 points (5-min UC segments). The criterion for the selection of the threshold parameter $\mathrm{r}$ proposed in $\mathrm{Lu}$ et al [16] was also considered. Entropy indices have been associated to complex cortical nervous system activity [15].

For each UC index, the difference between the last two hours of labor $\left(\mathrm{H}_{1}\right.$ and $\left.\mathrm{H}_{2}\right)$ and between the Normal and Operative groups was evaluated using the Spearman correlation coefficient, $95 \%$ bootstrap $(\mathrm{B}=1000)$ percentile confidence intervals $(95 \% \mathrm{CI})$ [17] for the median and nonparametric Mann-Whitney and Wilcoxon statistical tests [18], setting significance at $\mathrm{p}<0.05$.

\section{Results}

Progression of labor was associated with a significant increase of most UC linear (time- and frequency-domain) indices, both in normal and operative delivery groups (Table 2). The exception was the $\mathrm{HF}_{\text {norm }}$ index, which exhibited a significant decrease from $\mathrm{H}_{1}$ to $\mathrm{H}_{2}$ in both groups (Table 2), denoting a significant decrease of the respiratory frequency range. On the other hand, a significant increase of most entropy indices was observed in the normal group when the parameter $r$ was equal to $0.1,0.15$ or 0.2 , but the opposite was verified for the operative group considering $r_{\mathrm{Lu}}$ (Table 2). The temporal evolution of II, LF/HF and Sam$\mathrm{pEn}\left(2, \mathrm{r}_{\mathrm{Lu}}\right)$ is illustrated in Figure 1.

With respect to the comparison between the normal and operative delivery groups, II and most entropy indices were significantly lower in the normal group in $\mathrm{H}_{1}$, as well as for $\mathrm{HF}, \mathrm{HF}_{\text {norm }}$ and SD1 in $\mathrm{H}_{2}$ (Table 2 and Figure 1). The spectral indices $\mathrm{LF}_{\text {norm }}$ and $\mathrm{LF} / \mathrm{HF}$ were significantly higher in the normal delivery group during $\mathrm{H}_{2}$, probably associated with an increase of the sympatho-vagal more detectable in this group (Table 2 and Figure 1). 